\title{
BAER SUMS OF SPECIAL SCHREIER EXTENSIONS OF MONOIDS
}

\author{
NELSON MARTINS-FERREIRA, ANDREA MONTOLI, AND MANUELA SOBRAL
}

\begin{abstract}
We show that the special Schreier extensions of monoids, with abelian kernel, admit a Baer sum construction, which generalizes the classical one for group extensions with abelian kernel. In order to do that, we characterize the special Schreier extensions by means of factor sets.
\end{abstract}

\section{INTRODUCTION}

It is a well-known fact that, in the category of abelian groups, every set $\operatorname{Ext}(B, X)$ of isomorphic classes of extensions of an abelian group $B$ by an abelian group $X$ is endowed with an abelian group structure, called the Baer sum of extensions. This abelian group structure is related with cohomology: indeed, the $\operatorname{group} \operatorname{Ext}(B, X)$ is isomorphic to the second cohomology group of $B$ with coefficients in $X$. The same fact is true, more generally, in every category of $R$-modules, where $R$ is a commutative ring with unit.

In the category of groups the situation is more complicated. In fact, even when $X$ is an abelian group, it is not possible to endow $\operatorname{Ext}(B, X)$ with an abelian group structure which turns it isomorphic to a cohomology group. In order to have such a result, it is necessary to make a partition of the set $\operatorname{Ext}(B, X)$ into subsets $\operatorname{Ext}(B, X, \varphi)$ of isomorphic classes of extensions of $B$ by $X$ which induce a fixed action $\varphi: B \rightarrow \operatorname{Aut}(X)$ of $B$ on $X$ (see, for example, [4] for more details). Any of these subsets can be endowed with an abelian group structure (called again Baer sum) which turns it isomorphic to the second cohomology group of $B$ with coefficients in the $B$-module $\varphi: B \rightarrow \operatorname{Aut}(X)$.

The aim of the present paper is to describe a Baer sum construction for extensions of monoids. The extensions which allow such kind of construction are the so-called special Schreier extensions [1] with abelian kernel. The notion of special Schreier extension derives from the one of Schreier split epimorphism. This latter one has been introduced in [7] (although it appears implicitly already in [9]), and further developed in $[2,1]$. The notion of Schreier split epimorphisms of monoids is important because it is equivalent to the classical notion of monoid action, where an action of a monoid $B$ on a monoid $X$ is a monoid homomorphism $\varphi: B \rightarrow \operatorname{End}(X)$, $\operatorname{End}(X)$ being the monoid of endomorphisms of $X$. Special Schreier extensions are then those surjective homomorphisms such that their kernel congruence gives rise

Date: February 4, 2016.

2010 Mathematics Subject Classification. 20M32, 20M50, 20J99, 18 G50.

Key words and phrases. monoid, special Schreier extension, Baer sum, factor set.

This work was partially supported by the Centre for Mathematics of the University of Coimbra - UID/MAT/00324/2013, funded by the Portuguese Government through FCT/MEC and cofunded by the European Regional Development Fund through the Partnership Agreement PT2020, and also by ESTG and CDRSP from the Polytechnical Institute of Leiria.

The second author is a Postdoctoral Researcher of the Fonds de la Recherche ScientifiqueFNRS. 
to two Schreier split epimorphisms (all the needed details about these notions are recalled in Section 2).

The existence of a Baer sum construction for special Schreier extensions with abelian kernel was deduced in [1] by categorical arguments. Our goal here is to give a concrete and explicit description of the Baer sum. We show that, with every special Schreier extension with abelian kernel, it is possible to associate a factor set, similarly to what happens for group extensions. Our main result, Theorem 3.7, says that, given a monoid $B$, an abelian group $X$ and an action $\varphi: B \rightarrow \operatorname{End}(X)$ of $B$ on $X$, the set $\operatorname{SExt}(B, X, \varphi)$ of isomorphic classes of special Schreier extensions of $B$ by $X$ inducing the action $\varphi$ is in bijection with the quotient of the abelian group of factor sets by the subgroup of the so-called inner factor sets. This fact allows to endow $\operatorname{SExt}(B, X, \varphi)$ with an abelian group structure, which is the generalization of the classical Baer sum for group extensions. The study of the relationship between this abelian group structure and the cohomology of monoids is material for a future work.

\section{Schreier split epimorphisms and special Schreier EXtensions}

In this section we recall from $[7,2,1]$ the notions of Schreier split epimorphism, Schreier congruence and special Schreier extension.

\subsection{Schreier split epimorphisms.}

Definition 2.1 ([7], Definition 2.6). A split epimorphism $A \underset{f}{\stackrel{s}{\leftrightarrows}} B$ of monoids is said to be a Schreier split epimorphism when, for any $a \in A$, there exists a unique $x$ in the kernel $\operatorname{Ker}(f)$ of $f$ such that $a=x \cdot s f(a)$.

In other terms, a Schreier split epimorphism is a split epimorphism $(A, B, f, s)$ equipped with a unique set-theoretical map $q: A \rightarrow \operatorname{Ker}(f)$, called the Schreier retraction of $(A, B, f, s)$, with the property that, for any $a \in A$, we have:

$$
a=q(a) \cdot s f(a) \text {. }
$$

The definition of Schreier split epimorphism of monoids was first implicitly considered in [9], in connection with the notion of Schreier internal category (although the name Schreier extension was already used, with a different meaning, in older papers, see for example [11]). In [7] it was not only made explicit, but also extended to a wider class of algebraic structures, called monoids with operations (the name is inspired by the analogous notion of groups with operations in the sense of Porter [10], see also [8]). This class includes, beyond monoids, also commutative monoids, semirings, semilattices with a bottom element, distributive lattices and several other examples (see [7] for more details). Later, in [6], the definition of Schreier split epimorphism was considered in the wider context of Jónsson-Tarski varieties, i.e. varieties (in the sense of universal algebra) whose corresponding theories contain a unique constant 0 and a binary operation + satisfying the equalities $0+x=x+0=x$ for any $x$. For the purposes of the present paper, we restrict our attention only to the case of monoids.

Proposition 2.2 ([2], Proposition 2.4). A split epimorphism $(A, B, f, s)$ is a Schreier split epimorphism if and only if there exists a set-theoretical map $q: A \rightarrow \operatorname{Ker}(f)$ such that:

$$
\begin{aligned}
q(a) \cdot s f(a) & =a \\
q(x \cdot s(b)) & =x
\end{aligned}
$$

for every $a \in A, x \in \operatorname{Ker}(f)$ and $b \in B$. 
We showed in [7] (see also Section 5.2 in [1]) that the Schreier split epimorphisms of monoids correspond to the monoid actions, where an action of a monoid $B$ on a monoid $X$ is just a monoid homomorphism $\varphi: B \rightarrow \operatorname{End}(X), \operatorname{End}(X)$ being the monoid of endomorphisms of $X$. Let us briefly recall how the equivalence is obtained. Given a Schreier split epimorphism $A \underset{f}{\stackrel{s}{\leftrightarrows}} B$ with kernel $X$, the corresponding action $\varphi: B \rightarrow \operatorname{End}(X)$ is given by

$$
\varphi(b)(x)=q(s(b) \cdot x) .
$$

Conversely, given an action $\varphi: B \rightarrow \operatorname{End}(X)$, we can build a Schreier split epimorphism $A \underset{f}{\stackrel{s}{\longleftrightarrow}} B$ where $A$ is the semidirect product of $B$ and $X$ w.r.t. $\varphi$ : it is the cartesian product $X \times B$, equipped with the following monoid operation:

$$
\left(x_{1}, b_{1}\right) \cdot\left(x_{2}, b_{2}\right)=\left(x_{1} \cdot \varphi\left(b_{1}\right)\left(x_{2}\right), b_{1} \cdot b_{2}\right) .
$$

The following properties of the Schreier retraction will be useful later.

Proposition 2.3 ([1], Proposition 2.1.5). Given a Schreier split epimorphism $(A, B, f, s)$, we have:
(a) $q(x)=x$ for every $x \in \operatorname{Ker}(f)$;
(b) qs is the null homomorphism, i.e. $q s(b)=1$ for every $b \in B$;
(c) $q(1)=1$;
(d) if $b \in B$ and $x \in \operatorname{Ker}(f)$, then $q(s(b) \cdot x) \cdot s(b)=s(b) \cdot x$;
(e) for every $a, a^{\prime} \in A q\left(a \cdot a^{\prime}\right)=q(a) \cdot q\left(s f(a) \cdot q\left(a^{\prime}\right)\right)$.

Proof. (a) it is a straighforward consequence of the second identity in Proposition 2.2.

(b) for $b \in B$ we have:

$$
s(b)=1 \cdot s f(s(b))
$$

and the uniqueness of $q$ gives that $q s(b)=1$ for every $b \in B$.

(c) obviously we have $1=1 \cdot s f(1)$.

(d) for any $b \in B$ and any $x \in \operatorname{Ker}(f)$ we have:

$$
\begin{gathered}
s(b) \cdot x=q(s(b) \cdot x) \cdot s f(s(b) \cdot x)= \\
=q(s(b) \cdot x) \cdot s f s(b) \cdot s f(x)=q(s(b) \cdot x) \cdot s(b) .
\end{gathered}
$$

(e) $q\left(a \cdot a^{\prime}\right)$ is the unique element of $\operatorname{Ker}(f)$ such that

$$
a \cdot a^{\prime}=q\left(a \cdot a^{\prime}\right) \cdot s f\left(a \cdot a^{\prime}\right)=q\left(a \cdot a^{\prime}\right) \cdot s f(a) \cdot s f\left(a^{\prime}\right),
$$

so it suffices to prove that

$$
q(a) \cdot q\left(s f(a) \cdot q\left(a^{\prime}\right)\right) \cdot s f(a) \cdot s f\left(a^{\prime}\right)=a \cdot a^{\prime} .
$$

By point (d), we have that

$$
q\left(s f(a) \cdot q\left(a^{\prime}\right)\right) \cdot s f(a)=s f(a) \cdot q\left(a^{\prime}\right)
$$

and hence

$$
q(a) \cdot q\left(s f(a) \cdot q\left(a^{\prime}\right)\right) \cdot s f(a) \cdot s f\left(a^{\prime}\right)=q(a) \cdot s f(a) \cdot q\left(a^{\prime}\right) \cdot s f\left(a^{\prime}\right)=a \cdot a^{\prime} .
$$


2.2. Schreier internal relations. We call internal relation on a monoid $A$ a relation $R$ which is compatible with the monoid operation. It is known that it can be described equivalently as a submonoid of the product $A \times A$. By considering the homomorphic inclusion

$$
R \longmapsto A \times A
$$

and by composing it with the two projections of the product, we get two parallel homomorphisms

$$
R \underset{r_{2}}{\stackrel{r_{1}}{\longrightarrow}} A
$$

that are the first and the second projection of the relation. More explicitly, denoting an element of $R$ by a pair $(x, y)$, such that $x$ and $y$ belong to $A$ and are linked by the relation $R$, we have that $r_{1}(x, y)=x$ and $r_{2}(x, y)=y$.

An internal relation is reflexive when $r_{1}$ and $r_{2}$ have a common section $\sigma: A \rightarrow R$. In the notation above, we have that $\sigma(a)=(a, a)$ for any $a \in A$.

Definition 2.4 ([2], Definition 5.1). An internal reflexive relation of monoids

$$
R \underset{r_{2}}{\stackrel{r_{1}}{\rightleftarrows} \underset{\longrightarrow}{\rightleftarrows}} A
$$

is a Schreier reflexive relation if the split epimorphism $\left(R, A, r_{1}, \sigma\right)$ is a Schreier one.

It is well known that, in a Malt'sev variety [5], every internal reflexive relation is a congruence. This is false for the variety of monoids. However, a partial version of this result can be recovered for Schreier reflexive relations:

Theorem 2.5 ([2], Theorem 5.5). Any Schreier reflexive relation is transitive. It is a congruence if and only if $\operatorname{Ker}\left(r_{1}\right)$ is a group.

A Schreier reflexive relation which is a congruence will be called a Schreier congruence. We will be particularly interested in a specific kind of congruences, the so-called kernel congruences: given a monoid homomorphism $f: A \rightarrow B$, the corresponding kernel congruence $\operatorname{Eq}(f)$ is defined by: $a_{1} \operatorname{Eq}(f) a_{2}$ if and only if $f\left(a_{1}\right)=f\left(a_{2}\right)$. We represent $\operatorname{Eq}(f)$ with the following diagram:

$$
\operatorname{Eq}(f) \underset{f_{2}}{\stackrel{f_{1}}{\underset{f\langle 1,1\rangle}{\longrightarrow}}} A,
$$

where $\langle 1,1\rangle$ is the diagonal of $A:\langle 1,1\rangle(a)=(a, a)$. Let us observe that, thanks to the symmetry of the relation, the split epimorphisms $\left(f_{1},\langle 1,1\rangle\right)$ and $\left(f_{2},\langle 1,1\rangle\right)$ are isomorphic. Hence, if one of the two is a Schreier split epimorphism, the other is such, too.

2.3. Special Schreier homomorphisms. We recall from $[1,3]$ the following notion:

Definition 2.6. A monoid homomorphism $f: A \rightarrow B$ is a special Schreier homomorphism if the kernel congruence $E q(f)$ is a Schreier congruence.

As a consequence of Theorem 2.5, we have that the kernel of a special Schreier homomorphism, which is isomorphic to the kernel of the projections $f_{1}, f_{2}: \operatorname{Eq}(f) \rightarrow A$, is a group. A Schreier split epimorphism is not always a special Schreier homomorphism: it happens if and only if its kernel is a group ([3], Proposition 6.9). 
In the rest of the paper we will restrict our attention to special Schreier surjective homomorphisms. We first recall the following fact:

Proposition 2.7 ([1], Proposition 7.1.3). Every special Schreier surjective homomorphism $f: A \rightarrow B$ is the cokernel of its kernel. In other terms, the following sequence is an extension of $B$ by $\operatorname{Ker}(f)$ :

$$
\operatorname{Ker}(f) \stackrel{k}{\longrightarrow} A \stackrel{f}{\longrightarrow} B .
$$

Thanks to the previous proposition, a special Schreier surjective homomorphism can be called a special Schreier extension. Let $f: A \rightarrow B$ be a special Schreier extension. Then the split epimorphism

$$
X \stackrel{\langle k, 0\rangle}{\longrightarrow} \operatorname{Eq}(f) \underset{f_{2}}{\stackrel{\langle 1,1\rangle}{\longrightarrow}} A,
$$

where $\langle k, 0\rangle$ is the morphism sending $x \in X$ to $(x, 1)$, is a Schreier split epimorphism. Combining Proposition 2.2 and Lemma 2.3 we get the following:

Corollary 2.8. Let $f: A \rightarrow B$ be a special Schreier extension. Denote by $X$ the kernel of $f$. Then there exists a (unique) map $q: E q(f) \rightarrow X$ which satisfies the following conditions, for every $a \in A,\left(a_{1}, a_{2}\right),\left(a_{1}^{\prime}, a_{2}^{\prime}\right) \in E q(f)$ and $x \in X$ :

(i) $q\left(a_{1}, a_{2}\right) \cdot a_{2}=a_{1}$;

(ii) $q(x \cdot a, a)=x$;

(iii) $q(a \cdot x, a) \cdot a=a \cdot x$;

(iv) $q\left(a_{1} \cdot a_{1}^{\prime}, a_{2} \cdot a_{2}^{\prime}\right)=q\left(a_{1}, a_{2}\right) \cdot q\left(a_{2} \cdot q\left(a_{1}^{\prime}, a_{2}^{\prime}\right), a_{2}\right)$.

Proof. The first two conditions are just the reformulation of the conditions of Proposition 2.2. Conditions (iii) and (iv) are the reformulation of Conditions (d) and (e) of Lemma 2.3, respectively.

\section{Baer sums of SPecial Schreier extensions With Abelian Kernel}

The aim of this section is to give a description of the Baer sum of special Schreier extensions of monoids with abelian kernel (i.e. whose kernel is an abelian group), in analogy with the Baer sums of group extensions. In the group-theoretical case, it is not possible to define the Baer sum in the whole set $\operatorname{Ext}(B, X)$, where $B$ and $X$ are groups and $X$ is abelian. It is necessary to make a partition of this set into the subsets $\operatorname{Ext}(B, X, \varphi)$, where $\varphi: B \rightarrow \operatorname{Aut}(X)$ is a fixed group action of $B$ on $X$, and $\operatorname{Ext}(B, X, \varphi)$ is the set of isomorphic classes of extensions of $B$ by $X$ which induce the action $\varphi$.

In the case of monoids we have to make a similar partition of the set $\operatorname{SExt}(B, X)$ of isomorphic classes of special Schreier extensions of a monoid $B$ by an abelian group $X$. We recall that the Short Five Lemma holds for special Schreier extensions ([1], Proposition 7.2.1). Hence, two special Schreier extensions of $B$ by $X$ are isomorphic if and only if there is a morphism between them, as it happens in the case of group extensions. In order to make the partition we need, we first have to recall how to associate, with any element of $\operatorname{SExt}(B, X)$, an action of $B$ on $X$. Let $f: A \rightarrow B$ be a special Schreier extension with abelian kernel $X$. As we observed in the previous section, the Schreier split epimorphism

$$
X \stackrel{\langle k, 0\rangle}{\longrightarrow} \operatorname{Eq}(f) \underset{f_{2}}{\stackrel{\langle 1,1\rangle}{\longleftarrow}} A
$$


determines a monoid action $\psi: A \rightarrow \operatorname{End}(X)$ of $A$ on $X$. We can then define an action $\varphi: B \rightarrow \operatorname{End}(X)$ of $B$ on $X$, which is given by

$\varphi(b)(x)=\psi(a)(x)=q((a, a) \cdot(x, 1))=q(a \cdot x, a) \quad$ for all $a \in A$ such that $f(a)=b$.

This action of $B$ on $X$ is well defined (i.e. it does not depend on the choice of the inverse images of the elements of $B$ ) because $X$ is an abelian group. Hence the set $\operatorname{SExt}(B, X)$ of isomorphic classes of special Schreier extensions of $B$ by $X$ can be partitioned into the subsets $\operatorname{SExt}(B, X, \varphi)$ of isomorphic classes of special Schreier extensions which induce the action $\varphi$. We will endow any of this subsets with an abelian group structure (the Baer sum). In order to do this, we will describe special Schreier extensions in terms of factor sets. We start by introducing the following definition, which is a generalization of the classical notion of factor sets for group extensions:

Definition 3.1. Given a monoid $B$, an abelian group $X$ and an action $\varphi: B \rightarrow \operatorname{End}(X)$ of $B$ on $X$, a factor set is a map $g: B \times B \rightarrow X$ which satisfies, for all $b, b_{1}, b_{2}, b_{3} \in B$, the following conditions:

(i) $g(b, 1)=g(1, b)=1$;

(ii) $g\left(b_{1}, b_{2}\right) \cdot g\left(b_{1} \cdot b_{2}, b_{3}\right)=\varphi\left(b_{1}\right)\left(g\left(b_{2}, b_{3}\right)\right) \cdot g\left(b_{1}, b_{2} \cdot b_{3}\right)$.

We observe that the conditions above are the necessary and sufficient conditions in order to have that the cartesian product $X \times B$, equipped with the following binary operation, is a monoid:

Let

$$
\left(x_{1}, b_{1}\right) \cdot\left(x_{2}, b_{2}\right)=\left(x_{1} \cdot \varphi\left(b_{1}\right)\left(x_{2}\right) \cdot g\left(b_{1}, b_{2}\right), b_{1} \cdot b_{2}\right) .
$$

be a special Schreier extension with abelian kernel. We can associate with it a factor set in the following way: let $s: B \rightarrow A$ be a set-theoretical section of $f$ (it exists, since $f$ is surjective). Let us choose $s$ such that $s(1)=1$. Then, for any $b_{1}, b_{2} \in B$ :

$$
f\left(s\left(b_{1}\right) \cdot s\left(b_{2}\right)\right)=b_{1} \cdot b_{2}=f\left(s\left(b_{1} \cdot b_{2}\right)\right) .
$$

Hence the pair $\left(s\left(b_{1}\right) \cdot s\left(b_{2}\right), s\left(b_{1} \cdot b_{2}\right)\right)$ belongs to $\mathrm{Eq}(f)$. Consider then the following diagram:

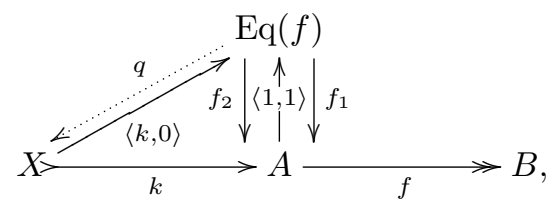

where $q$ is the Schreier retraction corresponding to the Schreier split epimorphism $\left(f_{2},\langle 1,1\rangle\right)$. Then we can define a map $g: B \times B \rightarrow X$ by putting:

$$
g\left(b_{1}, b_{2}\right)=q\left(s\left(b_{1}\right) \cdot s\left(b_{2}\right), s\left(b_{1} \cdot b_{2}\right)\right) .
$$

In order to prove that this map $g$ is a factor set, we need the following lemma:

Lemma 3.2. If $a_{1}, a_{2}, a_{3} \in A$ are such that $f\left(a_{1}\right)=f\left(a_{2}\right)=f\left(a_{3}\right)$, then

$$
q\left(a_{1}, a_{2}\right) \cdot q\left(a_{2}, a_{3}\right)=q\left(a_{1}, a_{3}\right) .
$$

Proof. By Condition (i) of Corollary 2.8, $q\left(a_{1}, a_{3}\right)$ is the unique element of $X$ such that $q\left(a_{1}, a_{3}\right) \cdot a_{3}=a_{1}$. Then it suffices to show that

$$
q\left(a_{1}, a_{2}\right) \cdot q\left(a_{2}, a_{3}\right) \cdot a_{3}=a_{1} .
$$

Using twice Condition (i) of Corollary 2.8, we get:

$$
q\left(a_{1}, a_{2}\right) \cdot q\left(a_{2}, a_{3}\right) \cdot a_{3}=q\left(a_{1}, a_{2}\right) \cdot a_{2}=a_{1} .
$$


Proposition 3.3. Given a special Schreier extension (2) with abelian kernel, the map $g$ defined as in (3) is a factor set.

Proof. The first factor set condition is easy to check, indeed for every $b \in B$ we have that:

$$
g(b, 1)=g(1, b)=q(s(b), s(b))=q\langle 1,1\rangle(s(b))=1,
$$

since $q\langle 1,1\rangle$ is the null homomorphism by Proposition 2.3 (b). It remains to prove that

$$
g\left(b_{1}, b_{2}\right) \cdot g\left(b_{1} \cdot b_{2}, b_{3}\right)=\varphi\left(b_{1}\right)\left(g\left(b_{2}, b_{3}\right)\right) \cdot g\left(b_{1}, b_{2} \cdot b_{3}\right)
$$

for all $b_{1}, b_{2}, b_{3} \in B$. The expression on the left is, by definition, equal to

$$
q\left(s\left(b_{1}\right) \cdot s\left(b_{2}\right), s\left(b_{1} \cdot b_{2}\right)\right) \cdot q\left(s\left(b_{1} \cdot b_{2}\right) \cdot s\left(b_{3}\right), s\left(b_{1} \cdot b_{2} \cdot b_{3}\right)\right) .
$$

Using Proposition $2.3(\mathrm{~b})$, we have that $q\left(s\left(b_{1} \cdot b_{2}\right), s\left(b_{1} \cdot b_{2}\right)\right)=1$. Hence the previous expression is equal to

$$
q\left(s\left(b_{1}\right) \cdot s\left(b_{2}\right), s\left(b_{1} \cdot b_{2}\right)\right) \cdot q\left(s\left(b_{1} \cdot b_{2}\right), s\left(b_{1} \cdot b_{2}\right)\right) \cdot q\left(s\left(b_{1} \cdot b_{2}\right) \cdot s\left(b_{3}\right), s\left(b_{1} \cdot b_{2} \cdot b_{3}\right)\right) .
$$

Using Corollary 2.8 (iv), this is the same as

$$
q\left(s\left(b_{1}\right) \cdot s\left(b_{2}\right) \cdot s\left(b_{3}\right), s\left(b_{1} \cdot b_{2}\right) \cdot s\left(b_{3}\right)\right) \cdot q\left(s\left(b_{1} \cdot b_{2}\right) \cdot s\left(b_{3}\right), s\left(b_{1} \cdot b_{2} \cdot b_{3}\right)\right)
$$

and, by Lemma 3.2 , this is $q\left(s\left(b_{1}\right) \cdot s\left(b_{2}\right) \cdot s\left(b_{3}\right), s\left(b_{1} \cdot b_{2} \cdot b_{3}\right)\right)$. Concerning the expression on the right, we have:

$$
\begin{gathered}
\varphi\left(b_{1}\right)\left(g\left(b_{2}, b_{3}\right)\right) \cdot g\left(b_{1}, b_{2} \cdot b_{3}\right)= \\
=\varphi\left(b_{1}\right)\left(q\left(s\left(b_{2}\right) \cdot s\left(b_{3}\right), s\left(b_{2} \cdot b_{3}\right)\right)\right) \cdot q\left(s\left(b_{1}\right) \cdot s\left(b_{2} \cdot b_{3}\right), s\left(b_{1} \cdot b_{2} \cdot b_{3}\right)\right)= \\
=q\left(s\left(b_{1}\right) \cdot q\left(s\left(b_{2}\right) \cdot s\left(b_{3}\right), s\left(b_{2} \cdot b_{3}\right)\right), s\left(b_{1}\right)\right) \cdot q\left(s\left(b_{1}\right) \cdot s\left(b_{2} \cdot b_{3}\right), s\left(b_{1} \cdot b_{2} \cdot b_{3}\right)\right) .
\end{gathered}
$$

Adding $q\left(s\left(b_{1}\right), s\left(b_{1}\right)\right)=1$ in the middle, we get

$q\left(s\left(b_{1}\right) \cdot q\left(s\left(b_{2}\right) \cdot s\left(b_{3}\right), s\left(b_{2} \cdot b_{3}\right)\right), s\left(b_{1}\right)\right) \cdot q\left(s\left(b_{1}\right), s\left(b_{1}\right)\right) \cdot q\left(s\left(b_{1}\right) \cdot s\left(b_{2} \cdot b_{3}\right), s\left(b_{1} \cdot b_{2} \cdot b_{3}\right)\right)$.

Using Corollary 2.8 (iv) and the fact that $X$ is commutative, we obtain that this expression is equal to

$$
q\left(s\left(b_{1}\right) \cdot s\left(b_{2}\right) \cdot s\left(b_{3}\right), s\left(b_{1}\right) \cdot s\left(b_{2} \cdot b_{3}\right)\right) \cdot q\left(s\left(b_{1}\right) \cdot s\left(b_{2} \cdot b_{3}\right), s\left(b_{1} \cdot b_{2} \cdot b_{3}\right)\right)
$$

and, using again Lemma 3.2 this is the same as $q\left(s\left(b_{1}\right) \cdot s\left(b_{2}\right) \cdot s\left(b_{3}\right), s\left(b_{1} \cdot b_{2} \cdot b_{3}\right)\right)$. So the two expressions are equal, and this concludes the proof.

Starting with a special Schreier extension (2) with abelian kernel, if we associate with it a factor set as we described before and we equip the cartesian product $X \times B$ with the monoid operation (1), we obtain an extension which is isomorphic to the one we started with:

Proposition 3.4. Given a special Schreier extension $X \stackrel{k}{\longrightarrow} A \stackrel{f}{\longrightarrow} B$ with abelian kernel $X$, define a factor set $g: B \times B \rightarrow X$ as in (3), where $s$ is a section of $f$ such that $s(1)=1$. Then we get isomorphisms of extensions as in the following diagram:

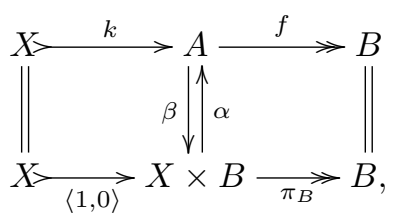

where the monoid operation on $X \times B$ is defined as in (1), $\pi_{B}$ is the product projection, and $\langle 1,0\rangle$ sends any $x \in X$ to $(x, 1)$. 
Proof. The map $\alpha: X \times B \rightarrow A$ is defined by

$$
\alpha(x, b)=x \cdot s(b)
$$

its inverse $\beta: A \rightarrow X \times B$ is given by

$$
\beta(a)=(q(a, s f(a)), f(a)) .
$$

These two maps are mutually inverse, indeed the pair $(a, s f(a))$ belongs to $\mathrm{Eq}(f)$; hence, thanks to the properties of the Schreier retraction $q$ (Proposition 2.2), we have that:

$$
(a, s f(a))=\langle k, 0\rangle q(a, s f(a)) \cdot\langle 1,1\rangle(s f(a))=(q(a, s f(a)), 1) \cdot(s f(a), s f(a)),
$$

and so

Conversely,

$$
a=q(a, s f(a)) \cdot s f(a)=\alpha \beta(a)
$$

$$
\beta \alpha(x, b)=\beta(x \cdot s(b))=(q(x \cdot s(b), s(b)), b),
$$

and we need to prove that $q(x \cdot s(b), s(b))=x$. But again, thanks to Proposition 2.2, we have that:

$$
q(x \cdot s(b), s(b))=q((x, 1) \cdot(s(b), s(b)))=q(\langle k, 0\rangle(x) \cdot\langle 1,1\rangle(s(b)))=x .
$$

Moreover, $\alpha$ (and then also $\beta$ ) is a monoid homomorphism. In order to show this, let us first recall that the monoid operation in $X \times B$ is given explicitly by:

$$
\left(x_{1}, b_{1}\right) \cdot\left(x_{2}, b_{2}\right)=\left(x_{1} \cdot \varphi\left(b_{1}\right)\left(x_{2}\right) \cdot q\left(s\left(b_{1}\right) \cdot s\left(b_{2}\right), s\left(b_{1} \cdot b_{2}\right)\right), b_{1} \cdot b_{2}\right),
$$

and $\varphi\left(b_{1}\right)\left(x_{2}\right)=q\left(s\left(b_{1}\right) \cdot x_{2}, s\left(b_{1}\right)\right)$. Hence

$$
\begin{gathered}
\alpha\left(\left(x_{1}, b_{1}\right) \cdot\left(x_{2}, b_{2}\right)\right)=\alpha\left(x_{1} \cdot q\left(s\left(b_{1}\right) \cdot x_{2}, s\left(b_{1}\right)\right) \cdot q\left(s\left(b_{1}\right) \cdot s\left(b_{2}\right), s\left(b_{1} \cdot b_{2}\right)\right), b_{1} \cdot b_{2}\right)= \\
=x_{1} \cdot q\left(s\left(b_{1}\right) \cdot x_{2}, s\left(b_{1}\right)\right) \cdot q\left(s\left(b_{1}\right) \cdot s\left(b_{2}\right), s\left(b_{1} \cdot b_{2}\right)\right) \cdot s\left(b_{1} \cdot b_{2}\right) .
\end{gathered}
$$

Using twice Corollary 2.8 (i), we obtain that the last expression is equal to:

$$
x_{1} \cdot q\left(s\left(b_{1}\right) \cdot x_{2}, s\left(b_{1}\right)\right) \cdot s\left(b_{1}\right) \cdot s\left(b_{2}\right)=x_{1} \cdot s\left(b_{1}\right) \cdot x_{2} \cdot s\left(b_{2}\right)=\alpha\left(x_{1}, b_{1}\right) \cdot \alpha\left(x_{2}, b_{2}\right) .
$$

Finally, it is immediate to see that $\alpha$ and $\beta$ are morphisms of extensions, i.e. that they make all the squares in Diagram (4) commute. This concludes the proof.

An immediate consequence of the previous proposition is the following fact, which will be useful later on:

Corollary 3.5. Given a special Schreier extension $X>k \rightarrow A \stackrel{f}{\longrightarrow} B$ with abelian kernel $X$, every $a \in A$ can be written in a unique way as $a=x \cdot s(b)$, with $x \in X$ and $b \in B$.

We described how we can associate a factor set $g$ with any special Schreier extension (2) with abelian kernel, by choosing a section $s$ of $f$. If we choose a different section $s^{\prime}$ of $f$, again with $s^{\prime}(1)=1$, we get another factor set $g^{\prime}$. Now we are interested in understanding the relationship between these two factor sets. So, let $s$ and $s^{\prime}$ be two chosen sections of $f$. Then, for every $b \in B, f s(b)=f s^{\prime}(b)$ and hence $\left(s^{\prime}(b), s(b)\right) \in \operatorname{Eq}(f)$. Thanks to the properties of the Schreier retraction $q$, we have that

$$
\left(s^{\prime}(b), s(b)\right)=\left(q\left(s^{\prime}(b), s(b)\right), 1\right) \cdot(s(b), s(b))
$$

and hence

$$
s^{\prime}(b)=q\left(s^{\prime}(b), s(b)\right) \cdot s(b) .
$$

Let us write $h(b)=q\left(s^{\prime}(b), s(b)\right)$. In this way we associate with the pair $\left(s, s^{\prime}\right)$ a map $h: B \rightarrow X$. Now, for any $b_{1}, b_{2} \in B$ we have, on one hand

$$
s^{\prime}\left(b_{1}\right) \cdot s^{\prime}\left(b_{2}\right)=h\left(b_{1}\right) \cdot s\left(b_{1}\right) \cdot h\left(b_{2}\right) \cdot s\left(b_{2}\right) .
$$


This last expression, by Corollary 2.8 (iii), is the same as

$$
\begin{gathered}
h\left(b_{1}\right) \cdot q\left(s\left(b_{1}\right) \cdot h\left(b_{2}\right), s\left(b_{1}\right)\right) \cdot s\left(b_{1}\right) \cdot s\left(b_{2}\right)= \\
=h\left(b_{1}\right) \cdot \varphi\left(b_{1}\right)\left(h\left(b_{2}\right)\right) \cdot s\left(b_{1}\right) \cdot s\left(b_{2}\right)= \\
=h\left(b_{1}\right) \cdot \varphi\left(b_{1}\right)\left(h\left(b_{2}\right)\right) \cdot g\left(b_{1}, b_{2}\right) \cdot s\left(b_{1} \cdot b_{2}\right),
\end{gathered}
$$

where the last equality holds because

$$
g\left(b_{1}, b_{2}\right) \cdot s\left(b_{1} \cdot b_{2}\right)=q\left(s\left(b_{1}\right) \cdot s\left(b_{2}\right), s\left(b_{1} \cdot b_{2}\right)\right) \cdot s\left(b_{1} \cdot b_{2}\right)=s\left(b_{1}\right) \cdot s\left(b_{2}\right)
$$

thanks to Corollary 2.8 (i).

On the other hand we have

$$
s^{\prime}\left(b_{1}\right) \cdot s^{\prime}\left(b_{2}\right)=g^{\prime}\left(b_{1}, b_{2}\right) \cdot s^{\prime}\left(b_{1} \cdot b_{2}\right)=g^{\prime}\left(b_{1}, b_{2}\right) \cdot h\left(b_{1} \cdot b_{2}\right) \cdot s\left(b_{1} \cdot b_{2}\right) .
$$

By comparing the two expressions, we obtain the equality

$$
h\left(b_{1}\right) \cdot \varphi\left(b_{1}\right)\left(h\left(b_{2}\right)\right) \cdot g\left(b_{1}, b_{2}\right) \cdot s\left(b_{1} \cdot b_{2}\right)=g^{\prime}\left(b_{1}, b_{2}\right) \cdot h\left(b_{1} \cdot b_{2}\right) \cdot s\left(b_{1} \cdot b_{2}\right) .
$$

Corollary 3.5 allows us to delete $s\left(b_{1} \cdot b_{2}\right)$ from both sides of the equality, obtaining the following one:

$$
h\left(b_{1}\right) \cdot \varphi\left(b_{1}\right)\left(h\left(b_{2}\right)\right) \cdot g\left(b_{1}, b_{2}\right)=g^{\prime}\left(b_{1}, b_{2}\right) \cdot h\left(b_{1} \cdot b_{2}\right) .
$$

Using the fact that $X$ is an abelian group, and that every element in the equality above belongs to $X$, we can rewrite the equality as follows:

$$
g^{\prime}\left(b_{1}, b_{2}\right) \cdot g\left(b_{1}, b_{2}\right)^{-1}=h\left(b_{1}\right) \cdot \varphi\left(b_{1}\right)\left(h\left(b_{2}\right)\right) \cdot h\left(b_{1} \cdot b_{2}\right)^{-1} .
$$

If, in particular, $f$ is a special Schreier split extension, i.e. there exists a section $s$ which is a monoid homomorphisms, then the factor set defined by means of this homomorphic section is the zero map. Hence the factor sets corresponding to special Schreier split epimorphisms are the following:

Definition 3.6. A factor set $g$ is called inner factor set if it is of the form

$$
g\left(b_{1}, b_{2}\right)=h\left(b_{1}\right) \cdot \varphi\left(b_{1}\right)\left(h\left(b_{2}\right)\right) \cdot h\left(b_{1} \cdot b_{2}\right)^{-1}
$$

for some map $h: B \rightarrow X$ such that $h(1)=1$.

We will denote by $\mathcal{F}(B, X, \varphi)$ the set of all the factor sets corresponding to a given action $\varphi: B \rightarrow \operatorname{End}(X)$, and by $\mathcal{J F}(B, X, \varphi)$ its subset of inner factor sets. The set of all functions $B \times B \rightarrow X$ has an abelian group structure inherited from the one of $X$. It is explicitly given by pointwise addition:

$$
\left(g_{1}+g_{2}\right)\left(b_{1}, b_{2}\right)=g_{1}\left(b_{1}, b_{2}\right) \cdot g_{2}\left(b_{1}, b_{2}\right) \text { for } g_{1}, g_{2}: B \times B \rightarrow X .
$$

It is not difficult to check that the set $\mathcal{F}(B, X, \varphi)$ is a subgroup of this group $X^{B \times B}$. The unique non-trivial condition to check is Condition (ii) of Definition 3.1, but it follows easily from the fact that $\varphi$ is a monoid homomorphism from $B$ to the monoid $\operatorname{End}(X)$ of endomorphisms of $X$. Similarly, it is easy to see that the subset $\mathcal{J F}(B, X, \varphi)$ of inner factor sets is a (normal) subgroup of $\mathcal{F}(B, X, \varphi)$.

All these facts, together with Proposition 3.4, give the following:

Theorem 3.7. The set $\operatorname{SExt}(B, X, \varphi)$ of isomorphic classes of special Schreier extensions of $a$ monoid $B$ by an abelian group $X$ inducing the action $\varphi: B \rightarrow \operatorname{End}(X)$ is in bijection with the factor abelian group

$$
\frac{\mathcal{F}(B, X, \varphi)}{\mathcal{J} \mathcal{F}(B, X, \varphi)} .
$$

Using this bijection, we can endow $\operatorname{SExt}(B, X, \varphi)$ with an abelian group structure, which is the Baer sum we were looking for. The unit of this abelian group is the isomorphic class of the split extension obtained by taking the semidirect product of $X$ and $B$ with respect to the action $\varphi$. 


\section{REFERENCES}

[1] D. Bourn, N. Martins-Ferreira, A. Montoli, M. Sobral, Schreier split epimorphisms in monoids and in semirings, Textos de Matemática (Série B), Departamento de Matemática da Universidade de Coimbra, vol. 45 (2013).

[2] D. Bourn, N. Martins-Ferreira, A. Montoli, M. Sobral, Schreier split epimorphisms between monoids, Semigroup Forum 88 (2014), 739-752.

[3] D. Bourn, N. Martins-Ferreira, A. Montoli, M. Sobral, Monoids and pointed S-protomodular categories, Homology, Homotopy and Applications, in press, preprint DMUC 14-04, 2014.

[4] S. Mac Lane, Homology, Springer-Verlag, 1963.

[5] A. I. Mal'cev, On the general theory of algebraic systems, Mat. Sbornik N. S. 35 (1954), 3-20.

[6] N. Martins-Ferreira, A. Montoli, On the "Smith is Huq" condition in S-protomodular categories, Appl. Categ. Structures, published online, DOI 10.1007/s10485-015-9411-1 (2015).

[7] N. Martins-Ferreira, A. Montoli, M. Sobral, Semidirect products and crossed modules in monoids with operations, J. Pure Appl. Algebra 217 (2013), 334-347.

[8] G. Orzech, Obstruction theory in algebraic categories I, J. Pure Appl. Algebra 2 (1972), 287-314.

[9] A. Patchkoria, Crossed semimodules and Schreier internal categories in the category of monoids, Georgian Math. Journal 5 (1998), n.6, 575-581.

[10] T. Porter, Extensions, crossed modules and internal categories in categories of groups with operations, Proceedings of the Edinburgh Math. Society 30 (1987), 373-381.

[11] L. Rédei, Die Verallgemeinerung der Schreierschen Erweiterungstheorie, Acta Sci. Math. Szeged 14 (1952), 252-273.

(Nelson Martins-Ferreira) ESTG, CDRSP, Instituto Politécnico de Leiria, Leiria, PorTUGAL

E-mail address: martins.ferreira@ipleiria.pt

(Andrea Montoli) CMUC, Universidade de Coimbra, 3001-501 Coimbra, Portugal AND

Institut de Recherche en Mathématique et Physique, Université catholique de Louvain, Chemin du CyClotron 2 Bte L7.01.02, 1348 Louvain-la-Neuve, Belgium

E-mail address: montoli@mat.uc.pt

(Manuela Sobral) CMUC and Departamento de Matemática, Universidade de Coimbra, 3001-501 Coimbra, Portugal

E-mail address: sobral@mat.uc.pt 